\title{
TM4SF5-CTD-2354A18.1-miR-4697-3P may play a key role in the pathogenesis of gastric cancer
}

\author{
Li DF ${ }^{1,2}$, Yang $\mathrm{MF}^{3}$, Shi $\mathrm{SL}^{1,2,4}$, Du YL ${ }^{1,2}$, Wang $\mathrm{H}^{1,2}$, Zhou YL ${ }^{1,2}$, Luo YL ${ }^{1,2}$, Ren $L^{5}$, Nie $\mathrm{YQ}^{1,2}$ \\ Department of Gastroenterology, Guangzhou Digestive Disease Center, Guangzhou First People’s Hospital, \\ Guangzhou Medical University, Guangzhou, China. nieyq@medmail.com.cn
}

\begin{abstract}
AIMS: Our aim is to identify important IncRNAs and mRNAs which may play a key role in contributing to pathogenesis of gastric cancer.

METHODS: Different LncRNAs and mRNAs are identified by microarray in gastric cancer tissue and corresponding normal tissues. The function and relationship of different LncRNAs and mRNAs is performed by GO analysis and Pathway analysis and made code-non-code network (CNC) by Pearson correlation coefficients (PCC). Then mRNA-miRNA relationship is predicted through mRNA-miRNA relationship software (http://www. targetscan.org). Lastly, mRNA-miRNA-LncRNA network is established for further research.

RESULTS: The expression profiles of 3732 IncRNAs showed different expression (fold change (FC) $\geq 2.0, p<$ 0.05 ) in gastric cancer tissue and normal tissue and expression profiles of 3994 mRNAs also showed different expression $(F C \geq 2.0, p<0.05)$ in gastric cancer and corresponding normal tissue.

CONCLUSION: The expression of TM4SF5, CTD-2354A18.1 and miR-4697-3P is in balance at physiological conditions, however, the balance is disrupted by some situations, which may contribute to gastric cancer. GO analysis and Pathway analysis also showed TM4SF5 played an important role in proliferation, differentiation and apoptosis. Therefore, TM4SF5-miR-4697-3P- CTD-2354A18.1 may play a key role in the pathogenesis of gastric cancer (Tab. 2, Fig. 4, Ref. 30). Text in PDF www.elis.sk. KEY WORDS: microarray, TM4SF5, miR-4697-3P, CTD-2354A18, gastric cancer
\end{abstract}

\section{Introduction}

Gastric cancer is a common malignant tumor, serious threat to human health and the third leading cause of cancer death (1). Because early diagnosis is very difficult, many of them are diagnosed at advanced stages with metastases (2). Therefore, it is an urgent issue to study the pathogenesis of gastric cancer and to find new effective markers of an early diagnosis of gastric cancer.

lncRNAs are greater than 200nt in length, lack coding potential, which were initially thought to be the product of a "noisy"

${ }^{1}$ Department of Gastroenterology, Guangzhou Digestive Disease Center, Guangzhou First People's Hospital, Guangzhou Medical University, Guangzhou, China, ${ }^{2}$ Guangzhou Key Laboratory of Digestive Disease, Guangzhou First People's Hospital, Guangzhou Medical University, Guangzhou, China, ${ }^{3}$ Department of Gastroenterology, Zhaoqing First People's Hospital, Zhaoqing, China, ${ }^{4}$ Department of Gastroenterology, Xiaolan People's Hospital, Zhaoqing, China, and ${ }^{5}$ Division of Gastroenterology and Hepatology, Ren JiHospital, School of Medicine, Sahnghai Jiao Tong University, Shanghai, China

Address for correspondence: YQ Nie, MD, Department of Gastroenterology, Guangzhou Digestive Disease Center, Guangzhou Key Laboratory of Digestive Disease, Guangzhou First People's Hospital, Guangzhou Medical University, No. 1 Panfu Road, Guangzhou 510180, China.

Phone: +86.20.81045975, Fax: +86.20.81045257

Acknowledgments: This work was supported by the National Natural Science Foundation of China (No. 81372633). The Doctoral Innovation Fund Projects from Shanghai Jiaotong University School of Medicine (BXJ201322) to Lin-Lin Ren. transcription of the genome with no biological function. However, recent studies have demonstrated that lncRNAs regulates several

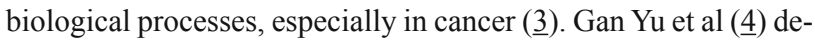
tected that clusters of lncRNAs were aberrantly expressed in renal clear cell carcinoma compared with corresponding normal tissue by microarray which may exert a partial or key role in tumor development. LEI HAN et al ( $\underline{5})$ indicated that lncRNA expression profile in glioblastoma (GBM) tissue was significantly altered by microarray, which may provide important insights into the mechanisms responsible for GBM progression and pathogenesis. Jin-ping Li et al (ㅁ) revealed different expression of lncRNAs in osteosarcoma compared with corresponding normal tissue and suggested that lncRNAs may be a novel candidate biomarker for the diagnosis of osteosarcoma and a potential target for therapy. Fu Yang et al (ㄱ) indicated that lncRNA-HEIH was an oncogenic lncRNA that promotes tumor progression and may serve as key regulatory hub in hepatic cellular cancer progression. Yong Yang et al ( $\underline{8})$ suggested that cisplatin resistance in non-small cell lung cancer cells may relate to the changes in noncoding RNAs, among these, LncRNA AK126698 appears to confer cisplatin resistance by targeting the Wnt pathway. Ji-hang Yuan et al (ㅁ) observed that the IncRNA-activated by TGF- $\beta$ (IncRNA-ATB) was up-regulated in hepatocellular carcinoma (HCC) metastases and associated with poor prognosis. Wei-Jun Cao et al (10) identified a set of lncRNAs differentially expressed in gastric cancer by microarray. Qianqian Pang et al (11) showed that the expression level of LINC00152 in gastric carcinoma was significantly increased, compared with 
matched normal tissue and normal mucosa from healthy controls. Weiliang Sun et al (12) showed that AC096655.1-002 was significantly down-regulated in gastric cancer tissues compared with paired adjacent non-tumorous tissues. Its expression level was significantly correlated with lymph node metastases, distant metastases, tumor-node-metastasis stages, and differentiation. Feng Yang et al (13) found that lncRNA gastric carcinoma highly expressed transcript 1 (IncRNA-GHET1) was up-regulated in gastric carcinoma, the over-expression of this lncRNA correlates with the tumor size, tumor invasion and poor survival. Ming Sun et al (14) found that MEG3 levels were markedly decreased in gastric cancer tissues compared with adjacent normal tissues, its expression level significantly correlated with TNM stages, depth of invasion, and tumor size. Therefore, LncRNA may closely relate to tumorigenesis, transfer, drug resistance and so on. Although it was found that the occurrence of gastric cancer closely correlated with the most of LncRNA, the functions of most of LncRNA are still unknown.

Central dogma of molecular biology suggests that genetic information is transferred from DNA to mRNA, then from mRNA to protein, thus, complete genetic information is transferred in the process of transcription and translation. Iit was proposed that the mRNAs seemed to have only one function, namely encoding protein (15). But, Poliseno (16) et al suggested that mRNAs not only regulate coding gene expression, but also reveal a non-coding function. Whitehead et al( 17$)$ found that DNA transcription not only encoded protein, but launched a divergent transcription generating the corresponding long non-coding RNA(LncRNA), then the divergently transcribed lncRNA/mRNA gene pairs exhibited coordinated changes in transcription when embryonic stem cells were differentiated into endoderm and revealed that transcription of most lncRNA genes were coordinated with transcription of protein-coding genes. The above studies of 1 ncRNA/mRNA gene pairs should provide new insights into the contributions of lncRNAs to the control of cell state and the process of differentiation.

MicroRNAs (miRNAs) are endogenous $~ 23$-nt RNAs that can play important gene-regulatory roles in animals and plants by pairing with the mRNAs of protein-coding genes to direct their posttranscriptional repression, they can be complementary to target mRNA 3'UTR in seed area (5' 2-7 bases), rely on Ago protein to decompose mRNA or deter mRNA translation ( $\underline{18})$. MiRNAs participated in the vast majority of biological processes, such as proliferation, differentiation and apoptosis and so on, and closely related to the occurrence of cancer such as gastric cancer (19). Hansen TB et al (20) found miRNA not only typically bound to the 3' UTR of target mRNAs, resulting in mRNA destabilization and translational repression, but also regulating gene expression by targeting non-coding antisense transcripts in human cells. They also discovered miR-671 can combine with LncRNAs, which was observed in the Cerebellar Degeneration-Related protein 1 (CDR1) locus, degrading the LncRNAs and decreasing the expression of CDR1 mRNA. MiRNAs can not only directly or indirectly regulate the expression of LncRNA, but in turn LncRNAs can also regulate the expression of miRNAs through sponge effect, which is known as competing endogenous RNA (ceRNA) (21). Jiayi Wang et al.(22) discovered CREB up-regulates long non-coding RNA, highly up-regulated in liver cancer (HULC) expression through interaction with microRNA-372 in liver cancer. Thus, LncRNA, mRNA, miRNA may play an important role in the pathogenesis of carcinoma.

Based on the above mentioned, we have studied LncRNAs and mRNAs expression profiles in gastric cancer tissues and corresponding normal tissues by microarray and evaluated the function and relationship of different LncRNAs and mRNAs by GO analysis, Pathway analysis and construct code-non-code network (CNC) to provide the theoretical foundation for pathogenesis of gastric cancer.

\section{Materials and methods}

\section{Patients and samples collection}

A total of 12 specimens were obtained from the Guangzhou First people's Hospital, China, from January 2013 to July 2013. Gastric cancer tissues and corresponding normal tissues which were $5 \mathrm{~cm}$ from the edge of cancer tissue, were obtained by surgical operation. All tissues were preserved in liquid nitrogen until used. There was no radiotherapy or chemotherapy applied prior to the operation. The study was approved by the Ethical Committee of Guangzhou First people's Hospital.

\section{RNA extraction}

Total RNA was extracted from tissues using the Trizol reagent (Invitrogen, UAS) according to the manufacturer's instructions. RNA quantity and quality was measured by NanoDrop ND-1000 and RNA integrity was assessed by standard denaturing agarose gel electrophoresis. Total RNA was eluted with RNA-se-free water and stored at $-80^{\circ} \mathrm{C}$.

\section{DN3A microarray}

Arraystar Human LncRNA Microarray V3.0 was designed for the global profiling of human LncRNAs and mRNAs, which was updated from the previous Microarray V2.0. About 30,586 LncRNAs and 26,109 mRNAs can be detected by third-generation LncRNA microarray. Data come from authorized data sources including NCBI RefSeq, UCSC, RNAdb.

\section{RNA labeling and array hybridization}

Sample labeling and array hybridization were performed according to the Agilent One-Color Microarray-Based Gene Expression Analysis protocol (Agilent Technology) with minor modifications. Briefly, mRNA was purified from total RNA after removal of rRNA (mRNA-ONLYTM Eukaryotic mRNA Isolation Kit, Epicentre). Then, each sample was amplified and transcribed into fluorescent cRNA along the entire length of the transcripts without 3' bias utilizing a random priming method. The labeled cRNAs were purified by RNeasy Mini Kit (Qiagen). The concentration and specific activity of the labeled cRNAs (pmol Cy3/ $\mu \mathrm{g}$ cRNA) were measured by NanoDrop ND-1000. $1 \mu \mathrm{g}$ of each labeled cRNA was fragmented by adding $5 \mu 110 \times$ Blocking Agent and $1 \mu \mathrm{l}$ of $25 \times$ Fragmentation Buffer, then the mixture was heated at $60^{\circ} \mathrm{C}$ 


\section{$608-615$}

for 30 min, finally $25 \mu 12 \times$ GE Hybridization buffer was added to dilute the labeled cRNA. $50 \mu$ of hybridization solution was dispensed into the gasket slide and assembled to the LncRNA and mRNA expression microarray slide. The slides were incubated for 17 hours at $65^{\circ} \mathrm{C}$ in an Agilent Hybridization Oven. The hybridized arrays were washed, fixed and scanned using the Agilent DNA Microarray Scanner (part number G2505C).

\section{Data analysis}

Agilent Feature Extraction software (version 11.0.1.1) was used to analyze the acquired array images. Quantile normalization and subsequent data processing were performed using the GeneSpring GX v12.0 software package (Agilent Technologies). After quantile normalization of the raw data, LncRNAs and mRNAs that had flags in Present or Marginal ("All Targets Value") in at least 6 out of 12 samples were chosen for further data analysis. Differentially expressed LncRNAs and mRNAs with statistical significance between the two groups were identified through Volcano Plot filtering. Hierarchical Clustering was performed using the R software (version 2.15). GO analysis and Pathway analysis were performed in the standard enrichment computation method.

\section{Disclosure of IncRNA-mRNA regulatory network}

In order to show that the lncRNAs directly regulated the expression of target mRNAs, we overlapped lncRNA targeted predictor onto the lncRNA-mRNA correlation network to construct regulatory network.

\section{Prediction of $m R N A-m i R N A$ regulation}

By using prediction of mRNA-miRNA network (http://www. targetscan.org), we depicted an mRNA-miRNA network.

\section{Depiction of miRNA-LncRNA-mRNA network}

According to the relationship between lncRNA, miRNA and mRNA, we constructed miRNA-LncRNA-mRNA network.

\section{Statistical analysis}

All statistical data were analyzed by SPSS 18.0 software (SPSS, Chicago, IL). Statistical analysis was done by Student's t-test. False discover rate (FDR) was calculated to correct the $\mathrm{p}$ value. The threshold values expressing different LncRNAs and mRNAs were a fold change $(\mathrm{FC} \geq 2.0, \mathrm{p}<0.05)$.

\section{Results}

1. The integrity of RNA can be assessed by electrophoresis on a denaturing agarose gel. Intact total RNA run on a denaturing gel will have sharp 28S and 18S rRNA bands (eukaryotic samples). The 28S rRNA band should be approximately twice as intense as the 18S rRNA band. This 2:1 intensity ratio indicates that the RNA is intact (Fig. 1a). Arraystar Human LncRNA Microarray V3.0 was designed for the global profiling of human LncRNAs and proteincoding transcripts, which was updated from the previous Microarray V2.0. About 30,586 LncRNAs and 26,109 coding transcripts can be detected by our third-generation microarray (Fig. 1b).
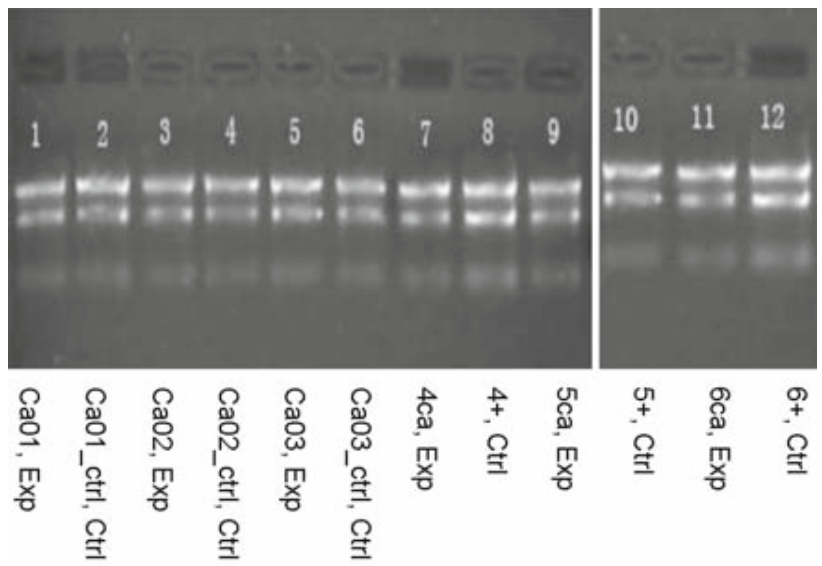

a

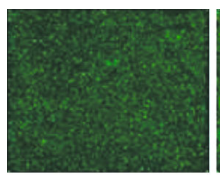

Ca01, Exp

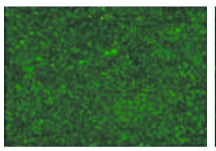

Ca03, Exp

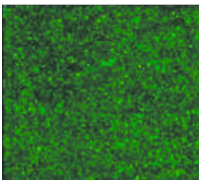

5Ca, Exp

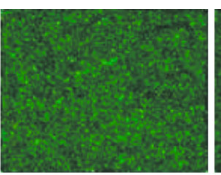

Ca01_ctrl, Ctrl

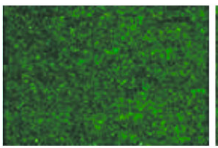

Ca03_ctrl, Ctrl

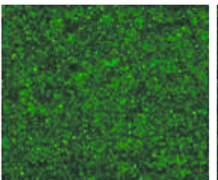

$5+$, Ctrl

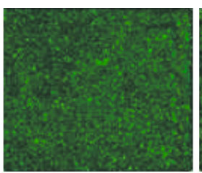

$\mathrm{CaO} 2$, Exp

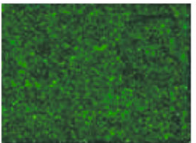

4Ca, Exp

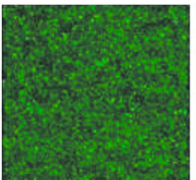

6Ca, Exp

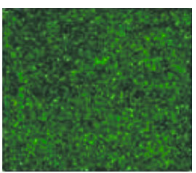

CaO2_ctrl, Ctrl

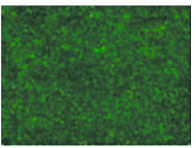

$4+$, Ctrl

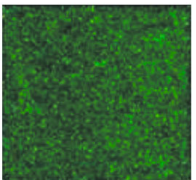

$6+$, Ctrl
Fig. 1. the quantity of RNA in each group (a), IncRNA and mRNA of microarray (b).

2. LncRNAs and mRNAs are aberrantly expressed in gastric cancer compared with corresponding normal tissue.

We determined lncRNAs and mRNAs expression profiles in gastric cancer by microarray. The boxplot view was used to look at, and compare, the distributions of expression values for the samples in an experiment after normalization (Fig. 2a, 2d). After normalization, the scatter plot was a visualization that was useful for assessing the variation between gastric cancer tissue and corresponding normal tissue (Fig. 2b, 2e). Hierarchical Clustering showed distinguishable expression profiles of lncRNAs and mRNAs between gastric cancer tissue and corresponding normal tissue. "Red" showed high relative expression, "blue" showed low relative expression (Fig. 2c, 2f). There were expression profiles of 3732 lncRNAs determined to be different $(F C \geq 2.0, p<0.05)$, among these, there were 2451 lncRNAs which were identified to be up-regulated more than two-fold, 8 lncRNAs which were identified to be up-regulated more than forty-fold, however there were 1281 lncRNAs which were down-regulated more than twofold, 9 lncRNAs which were down-regulated more than forty-fold 
a

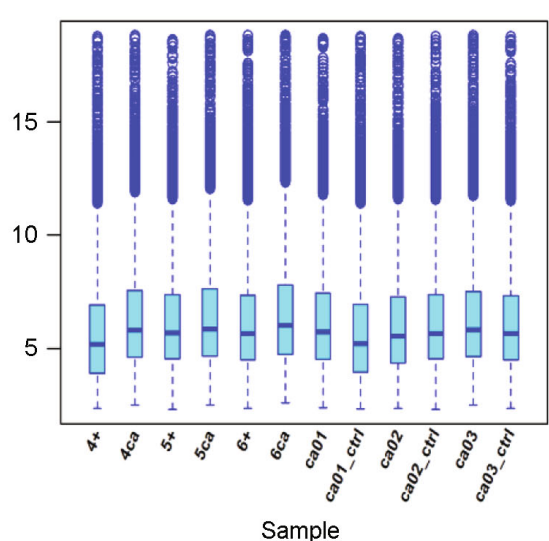

(C)

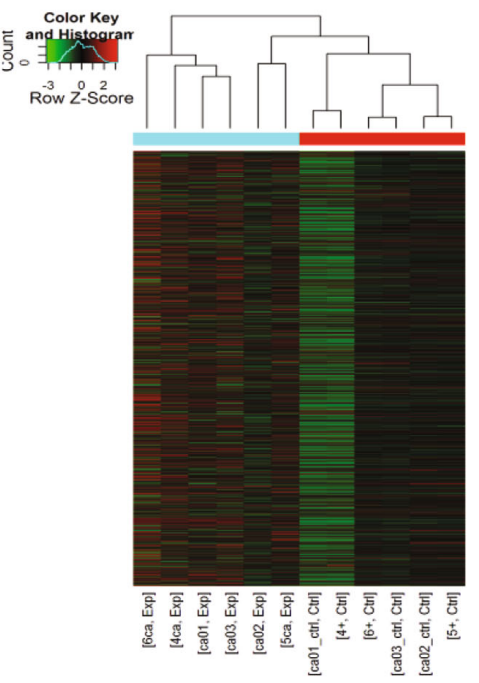

e

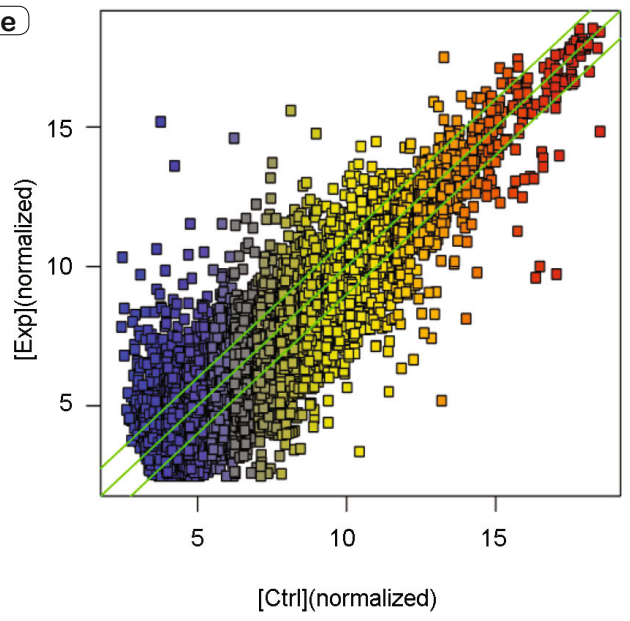

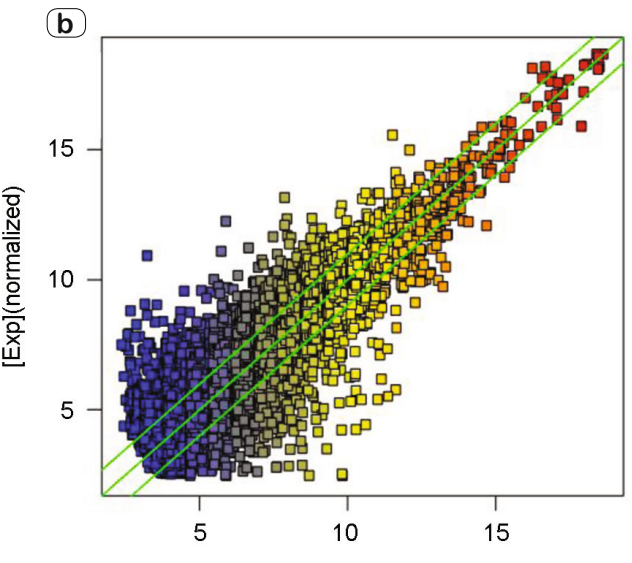

[Ctrl](normalized)

(d)

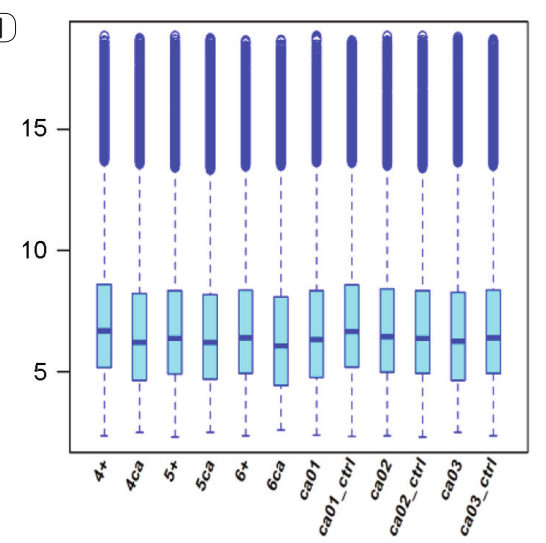

Sample

(f)

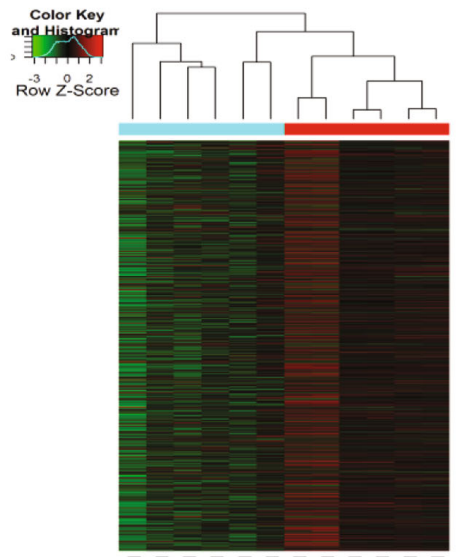

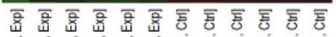

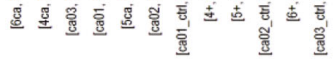

Fig. 2. LncRNAs and mRNAs profile comparison between gastric cancer tissue and corresponding normal tissue. The box plot is a traditional method for visualizing the distribution of a dataset in the IncRNA and mRNA profiles (a, d). After normalization, the distributions of log2ratios among the tested samples are nearly the same. The scatter plot is a visualization that is useful for assessing the variation between the gastric cancer tissue and the corresponding normal tissue (b, e). Hierarchical Clustering shows distinguishable expression profiling of IncRNAs and mRNAs between the gastric cancer tissue and the corresponding normal tissue. "Red" shows high relative expression, "blue" shows low relative expression (c, f). 
608-615

Tab. 1. The different expression profiles of LncRNAs.

\begin{tabular}{|c|c|c|c|c|c|}
\hline ProbeName & $\mathrm{p}$ & $\mathrm{FC}$ & Regulation & seqname & GeneSymbol \\
\hline ASHGA5P032547 & 4.17072E-08 & 207.6425 & up & uc010wmx.2 & LINC00483 \\
\hline ASHGA5P048470 & 3.86443E-05 & 108.85076 & up & TCONS 00013751 & XLOC 006390 \\
\hline ASHGA5P042490 & 7.98541E-05 & 81.69372 & up & ENST00000418403 & RP11-462G2.1 \\
\hline ASHGA5P044202 & 4.13695E-06 & 70.630486 & up & ENST00000519691 & RP11-150012.3 \\
\hline ASHGA5P035008 & $2.00358 \mathrm{E}-05$ & 44.67928 & up & ENST00000444871 & RP11-89K21.1 \\
\hline ASHGA5P027435 & $1.60304 \mathrm{E}-05$ & 42.6102 & up & NR_026836 & TRHDE-AS1 \\
\hline ASHGA5P028749 & 0.000363809 & 42.228905 & up & ENST00̄000567234 & LINC00543 \\
\hline ASHGA5P033892 & $1.28979 \mathrm{E}-17$ & 163.69966 & down & ENST00000577634 & CTD-2354A18.1 \\
\hline ASHGA5P044551 & 4.69033E-12 & 154.65607 & down & ENST00000441160 & RP11-400N13.2 \\
\hline ASHGA5P047944 & $1.18041 \mathrm{E}-10$ & 74.84269 & down & ENST00000532065 & RP11-231N3.1 \\
\hline ASHGA5P025715 & 0.000277271 & 71.95296 & down & NR_027910 & CALB2 \\
\hline ASHGA5P053278 & $3.65474 \mathrm{E}-07$ & 65.5398 & down & ENST000000439070 & AC002066.1 \\
\hline ASHGA5P015469 & 0.001119005 & 57.994392 & down & ENST00000577817 & AC006050.3 \\
\hline ASHGA5P023451 & $2.03083 \mathrm{E}-05$ & 57.51053 & down & ENST00000581210 & KRT16P1 \\
\hline ASHGA5P023350 & 1.96179E-07 & 49.086155 & down & ENST00000579062 & KRT16P2 \\
\hline
\end{tabular}

The different expression profiles of lncRNAs between gastric cancer and corresponding normal tissue (FC $\geq 40.0, p<0.05)$.

\begin{tabular}{|c|c|c|c|c|c|}
\hline ProbeName & $\mathrm{p}$ & $\mathrm{FC}$ & Regulation & seqname & GeneSymbol \\
\hline ASHGA5P004644 & $1.68972 \mathrm{E}-05$ & 2742.892 & up & NM_001443 & FABP1 \\
\hline ASHGA5P043129 & $2.08182 \mathrm{E}-07$ & 664.3489 & up & NM_001306 & CLDN3 \\
\hline ASHGA5P048641 & 7.79122E-06 & 331.62485 & up & NM_0̄01145643 & PHGR1 \\
\hline ASHGA5P009276 & $4.45973 \mathrm{E}-07$ & 229.73157 & up & NM_032965 & CCL15 \\
\hline ASHGA5P034190 & $2.31954 \mathrm{E}-06$ & 176.69968 & up & NM_006149 & LGALS4 \\
\hline ASHGA5P003560 & $1.61705 \mathrm{E}-06$ & 127.98858 & up & ENST00̄000270560 & TM4SF5 \\
\hline ASHGA5P031401 & 0.001050582 & 108.84058 & up & NM_019062 & RNF186 \\
\hline ASHGA5P005831 & $3.73523 \mathrm{E}-06$ & 74.06561 & up & NM_176813 & AGR3 \\
\hline ASHGA5P021813 & $7.55469 \mathrm{E}-06$ & 61.442944 & up & NM $\overline{00} 1195597$ & LOC 100507203 \\
\hline ASHGA5P053959 & $1.26144 \mathrm{E}-05$ & 60.98936 & up & NM_o00531 & OTC \\
\hline ASHGA5P002054 & $1.88854 \mathrm{E}-08$ & 255.58185 & down & ENST000000252250 & KRT6C \\
\hline ASHGA5P032422 & $5.47804 \mathrm{E}-10$ & 157.87292 & down & NM 000526 & KRT14 \\
\hline ASHGA5P033632 & $3.91441 \mathrm{E}-06$ & 135.8879 & down & ENST00000283752 & SERPINB3 \\
\hline ASHGA5P001587 & $1.19176 \mathrm{E}-06$ & 108.11994 & down & NM_000582 & SPP1 \\
\hline ASHGA5P054352 & $2.48634 \mathrm{E}-05$ & 60.232204 & down & NM_001354 & AKR1C2 \\
\hline
\end{tabular}

The different expression profiles of mRNAs between the gastric cancer tissue and the corresponding normal tissue $(\mathrm{FC} \geq 60.0, \mathrm{p}<0.05)$

(Fig. 2, Tab. 1). There were expression profiles of 3994 mRNAs determined to be different expression $(F C \geq 2.0, p<0.05)$, among these, there were $969 \mathrm{mRNAs}$ which were identified to be up-regulated more than two-fold, $11 \mathrm{mRNAs}$ which were identified to be up-regulated more than sixty-fold, however, there were 3025 mRNAs which were identified to be down-regulated more than two-fold, 6 mRNA which were down-regulated more than sixtyfold (Fig. 2, Tab. 2).

3. Construction of the coding-non-coding gene co-expression network (CNC network)

Pearson correlation coefficients (PCC) were calculated for important genes and significant correlation pairs were merged with each LncRNAs to investigate mRNAs and LncRNAs co-expression. It was used to calculate Pearson correlation coefficient between the coding and non - coding by R-Environment statistics. There was a correlation between mRNAs and LncRNAs (PCC $\geq$ 0.95) (Fig. 3a, 3b).

4. We found TM4SF5, which was over-expressed in gastric cancer (Tab. 2), which was also over-expressed in other kinds of cancer (23-26), so drew the CNC network between TM4SF5 and LncRNAs by PCC (Fig. 4a). It was to predicted miRNAs for TM4SF5 (http://www.targetscan.org), and has-miR-4697-3p was found - which can combine with TM4SF5 3'UTR (Fig. 4b). Lastly, we found the sequences of Linc00483, CTD-2354A18.1, RP11-150O1, miR-4697-3P (http://genome.ucsc.edu/), discovered there were more than three gene loci of CTD-2354A18.1, which can combine with miR-4697-3P (Fig. 4c).

5. GO analysis and pathway analysis

The Gene Ontology project provided a controlled vocabulary to describe gene and gene product attributes in any organism (http:// www.geneontology.org). The ontology covered three domains: Biological Process, Cellular Component and Molecular Function. The p-value denoted the significance of GO terms enrichment in the differential expression (DE) genes. The results indicated GO terms enrichment, especially, proliferation, differentiation and apoptosis which was related closely to cancer (Fig. 4). Based on the latest KEGG (http://www.genome.jp/kegg) database, we provided pathway analysis for differentially expressed mRNAs. The results indicated that there were 86 signaling pathways, many related to cell cycle, angiogenesis and so on. 


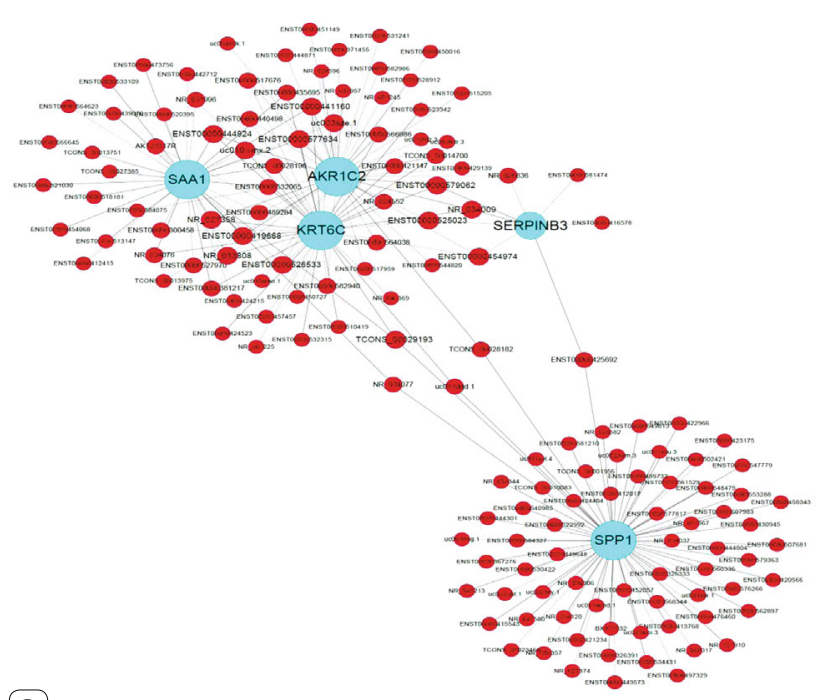

a

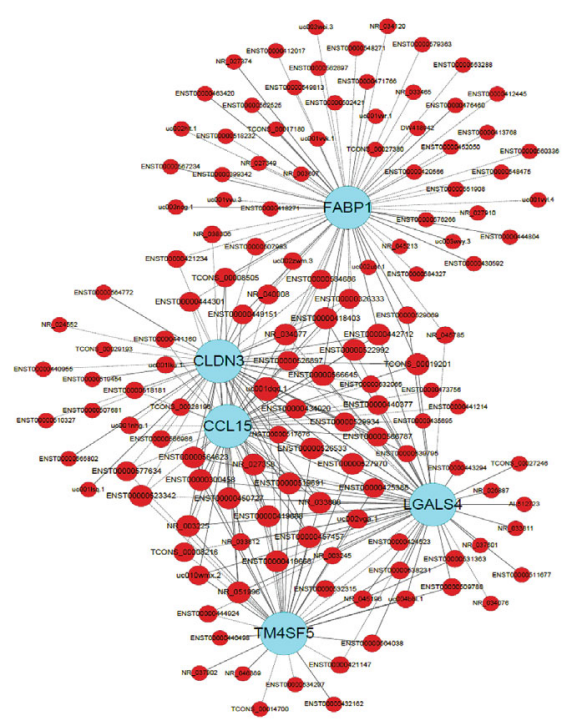

Fig. 3. mRNA-IncRNA association network, the solid line indicates positive correlation, dotted line indicates negative correlation $(a, b)$.

\section{Discussion}

Gastric cancer is the most common and aggressive type of primary gastrointestinal tumor in humans. In the past, the molecular mechanisms to gastric cancer have extensively been studied. But the pathogenesis of the disease is still unclear, and the gene regulation involved in the disease has been vague until now.

More and more evidence has proved lncRNAs and mRNAs, which may be the most important factors, to result in gastric cancer $(10,27,28)$. Therefore, we evaluated the lncRNAs and mRNAs expression profiles in gastric cancer tissue and corresponding non-tumorous tissues in patients, which was to reveal the potential role of lncRNAs and mRNAs in the pathogenesis of gastric cancer. Microarray techniques discovered a set of differentially ex- pressed lncRNAs and mRNAs, there were 2541 up-regulated and 1281 down-regulated lncRNAs, 969 up-regulated and 3025 downregulated mRNAs in gastric cancer tissue compared to normal tissue, respectively ( $F C \geq 2, p<0.05$ ). Among them, 8 lncRNAs which were identified to be up-regulated more than forty-fold, 9 lncRNAs which were down-regulated more than forty-fold, 11 mRNAs which were identified to be up-regulated more than sixtyfold, 6 mRNA which were down-regulated more than sixty-fold.

In order to predict the function of different lncRNAs and mRNAs, we constructed the coding-non-coding gene co-expression network by PCC. We discovered the mRNAs, such as FABP1, CLDN3, CCL15, LGALS4, TM4SF5, KRT6C, AKR1C2, SERPINB3 and so on, which were closely related to the abnormal LncRNAs, for example LINC00483, CTD-2354A18.1 and so on, especially between TM4SF5 and LINC00483, CTD-2354A18.1 $(\mathrm{PCC}=0.987922239060751, \mathrm{PCC}=-0.953951626881595)$.

According to endogenous RNA(ceRNA), we predicted miRNAs related to TM4SF5, and found has-miR-4697-3p which can combine with TM4SF5 in the ORF (http://www.targetscan.org). Next, we referred to the sequence of LINC00483, CTD-2354A18.1 and RP11-150O12.3, disclosed there were more than three gene loci of CTD-2354A18.1, which can combine with miR-4697-3P, however, LINC00483 and RP11-150O12.3 cannot (http://genome.ucsc.edu/).

Trans-membrane 4L six family member 5 (TM4SF5), as a membrane glycoprotein with 4 trans-membrane domains, is similar to the tetraspanins in terms of membrane topology and plays important roles in tumorigenesis and tumor metastasis (29). Yong-bing Wu et al (26) demonstrated TM4SF5 was more highly expressed in human esophageal cancer cells and human esophageal cancer tissues than in normal esophageal epithelial cells and matched non-tumor tissues, and considered a high level of TM4SF5 was associated with human esophageal cancer progression and poor patient survival. Sanghoon Kwon et al (25) suggested TM4SF5specific peptide vaccine had a therapeutic effect against colon cancer in a mouse model. Jihye Ryu et al (무) found TM4SF5 over-expressed in hepatocellular carcinoma activated focal adhesion kinase (FAK) during tumor cell migration, further discovered hepatic cancer cells adopt TM4SF5-dependent FAK activation and metastatic potential by lowering IL-6 expression and avoiding its immunological action through the IL-6-STAT3 pathway. Therefore, TM4SF5 relates closely to tumorigenesis. We discovered the over expression of TM4SF5 in gastric cancer tissue in patients. But why it is over-expression? Through our study, we think that it may be the disruption of TM4SF5, CTD-2354A18.1 and miR4697-3P. The expressions of TM4SF5, CTD-2354A18.1 and miR4697-3P keep balance in normal state, however, the balance was disrupted by some situation, which may decrease the expression of CTD-2354A18.1 resulting in a decrease of CTD-2354A18.1 to combine with miR-4697-3P, in contrast, more miR-4697-3P can combine with the ORF of TM4SF5, leading to over expression of TM4SF5, which may contribute to gastric cancer. GO term and KEGG pathway analysis also showed TM4SF5 may play an important role in proliferation, differentiation and apoptosis. In our future study, we will study the expression of TM4SF5, CTD2354A18.1 and miR-4697-3P in gastric cancer tissue and gastric 
$608-615$

(a)

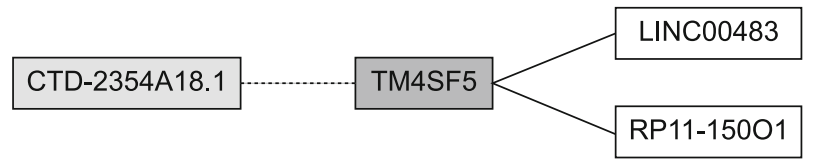

(b)

\begin{tabular}{|c|c|c|c|c|c|c|c|c|c|c|c|c|}
\hline & $\begin{array}{l}\text { predicted consequential pairing of } \\
\text { target region (top) and miRNA (bottom) }\end{array}$ & $\begin{array}{c}\text { seed } \\
\text { match }\end{array}$ & $\begin{array}{l}\text { site-type } \\
\text { contri- } \\
\text { bution }\end{array}$ & $\begin{array}{l}\text { 3'pairing } \\
\text { contri- } \\
\text { bution }\end{array}$ & $\begin{array}{l}\text { local AU } \\
\text { contri. } \\
\text { bution }\end{array}$ & $\begin{array}{l}\text { position } \\
\text { contri- } \\
\text { bution }\end{array}$ & $\begin{array}{c}\mathrm{TA} \\
\text { contribution }\end{array}$ & $\begin{array}{c}\text { SPS } \\
\text { contribution }\end{array}$ & $\begin{array}{l}\text { context+ } \\
\text { score }\end{array}$ & $\begin{array}{c}\text { context+ } \\
\text { score } \\
\text { percentile }\end{array}$ & $\begin{array}{c}\text { conserved } \\
\text { branch } \\
\text { length }\end{array}$ & PCI \\
\hline $\begin{array}{l}\text { Position 6-12 of TM4SF5 3' UTR } \\
\text { hsa-miR-4697-3p }\end{array}$ & 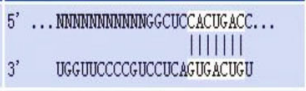 & $\begin{array}{c}7 \mathrm{mer}- \\
\mathrm{m} 8\end{array}$ & -0.120 & N/A & N/A & $N / A$ & $N / A$ & N/A & N/A & $\begin{array}{l}\text { too close } \\
\text { to ORF }\end{array}$ & 0.773 & N/A \\
\hline $\begin{array}{l}\text { Position } 14-20 \text { of TM4SF5 3' UTR } \\
\text { hsa-miR-941 }\end{array}$ & 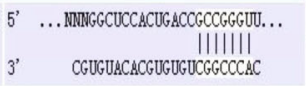 & $\begin{array}{l}7 \mathrm{mer}- \\
\mathrm{m} 8\end{array}$ & -0.120 & N/A & N/A & N/A & $N / A$ & NA & $N / A$ & $\begin{array}{l}\text { too close } \\
\text { to ORF }\end{array}$ & 0.009 & N/A \\
\hline $\begin{array}{l}\text { Position } 22-28 \text { of TM4SF5 } 3^{\prime} \text { UTR } \\
\text { hsa-miR-3934 }\end{array}$ & 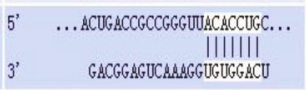 & $\begin{array}{l}7 \mathrm{mer}- \\
\mathrm{m8}\end{array}$ & -0.120 & 0.003 & 0.045 & -0.059 & 0.015 & -0.032 & -0.15 & 80 & 0.795 & $N / A$ \\
\hline $\begin{array}{l}\text { Position 23-29 of TM4SF5 3' UTR } \\
\text { hsa-miR-3665 }\end{array}$ & $\begin{array}{l}5^{\prime} \text {... CUGACCGCCGGGUUACACCUGCU... } \\
\text { 3' } \quad \|_{\text {GCGGCGGGGGUGGACGA }}\end{array}$ & $\begin{array}{c}7 \mathrm{mer}- \\
\mathrm{m} 8\end{array}$ & -0.120 & -0.035 & 0.021 & -0.059 & 0.012 & -0.068 & -0.25 & 89 & 1.009 & $N / A$ \\
\hline $\begin{array}{l}\text { Position 30-36 of TM4SF5 3' UTR } \\
\text { hsa-miR-4533 }\end{array}$ & 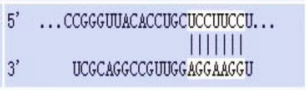 & $\begin{array}{c}7 \mathrm{mer}- \\
\mathrm{m} 8\end{array}$ & -0.120 & 0.012 & 0.089 & -0.058 & 0.030 & -0.036 & -0.08 & 52 & 0.009 & N/A \\
\hline $\begin{array}{l}\text { Position 31-37 of TM4SF5 3' UTR } \\
\text { hsa-miR-4476 }\end{array}$ & 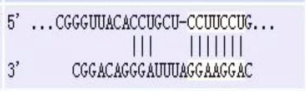 & $\begin{array}{l}7 \mathrm{mer}- \\
\mathrm{m} 8\end{array}$ & -0.120 & -0.016 & 0.061 & -0.057 & 0.030 & -0.030 & -0.13 & 75 & 0.009 & N/A \\
\hline $\begin{array}{l}\text { Position } 34-40 \text { of TM4SF5 } 3^{\prime} \text { UTR } \\
\text { hsa-miR-665 }\end{array}$ & $\begin{array}{l}5^{\prime} \text {...GUUACACCUGCUCCUUCCUGGAC... } \\
3^{\prime} \quad \||||||||| \mid\end{array}$ & $\begin{array}{c}7 \mathrm{mer}- \\
1 \mathrm{~A}\end{array}$ & -0.074 & -0.006 & 0.062 & -0.046 & 0.033 & -0.065 & -0.10 & 48 & 1.102 & N/A \\
\hline $\begin{array}{l}\text { Position } 70-76 \text { of TM4SF5 3' UTR } \\
\text { hsa-miR-330-3p }\end{array}$ & 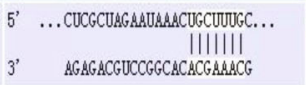 & $\begin{array}{c}7 \mathrm{mer}- \\
\mathrm{m} 8\end{array}$ & -0.120 & 0.003 & 0.052 & -0.061 & 0.020 & 0.023 & -0.08 & 61 & 0.724 & $N / A$ \\
\hline
\end{tabular}

(c)

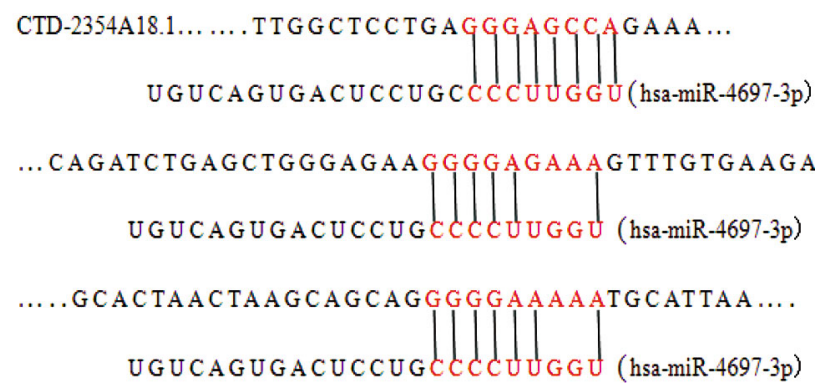

Fig. 4. TM4SF5 relates closely to Linc00483, CTD-2354A18.1, and RP11-15001 by PCC, the solid line indicates positive correlation, dotted line indicates negative correlation (a). It finds has-miR-4697-3p which can combine with in the ORF of TM4SF5 (http://www.targetscan.org) (b). It discloses there are more than three gene loci of CTD-2354A18.1, which can combine with miR-4697-3P (http://genome.ucsc.edu/) (c).

cancer cells. The relationship between TM4SF5, CTD-2354A18.1 and miR-4697-3P will be shown by transfection, immune-precipitation, flow-cytometric analysis of apoptosis, cell migration and invasion assays and so on, which may create a more detailed picture of the pathogenesis of gastric cancer.

\section{References}

1. Venerito M, Nardone G, Selgrad M, Rokkas T, Malfertheiner P. Gastric cancer-epidemiologic and clinical aspects. Helicobacter 2014; 19 (Suppl 1): 32-37. PMID: 25167943.

2. Asaka M, Mabe K. Strategies for eliminating death from gastric cancer in Japan. Proc Jap Acad Series B, Phys Biol Sci 2014; 90 (7): 251-258. PMID: 25169671.
3. Mathieu EL, Belhocine M, Dao LT, Puthier D, Spicuglia S. Functions of lncRNA in development and diseases. Medecine sciences : M/S. 2014; 30 (8-9): 790-6. PMID: 25174757. Role des longs ARN non codants dans le developpement normal et pathologique.

4. Yu G, Yao W, Wang J, Ma X, Xiao W et al. LncRNAs expression signatures of renal clear cell carcinoma revealed by microarray. PloS one 2012; 7 (8): e42377. PMID: 22879955. Central PMCID: 3412851.

5. Han L, Zhang K, Shi Z, Zhang J, Zhu J et al. LncRNA pro fi le of glioblastoma reveals the potential role of IncRNAs in contributing to glioblastoma pathogenesis. Intern J Oncol 2012; 40 (6): 2004-2012. PMID: 22446686.

6. Li JP, Liu LH, Li J, Chen Y, Jiang XW et al. Microarray expression profile of long noncoding RNAs in human osteosarcoma. Biochem Biophys Res Com 2013; 433 (2): 200-206. PMID: 23466354. 
7. Yang F, Zhang L, Huo XS, Yuan JH, Xu D et al. Long noncoding RNA high expression in hepatocellular carcinoma facilitates tumor growth through enhancer of zeste homolog 2 in humans. Hepatology 2011; 54 (5): 1679-1689. PMID: 21769904.

8. Yang Y, Li H, Hou S, Hu B, Liu J. The noncoding RNA expression profile and the effect of IncRNAAK126698 on cisplatin resistance in nonsmall-cell lung cancer cell. PloS one 2013; 8 (5): e65309. PMID: 23741487. Central PMCID: 3669360.

9. Yuan JH, Yang F, Wang F, Ma JZ, Guo YJ et al. A long noncoding RNA activated by TGF-beta promotes the invasion-metastasis cascade in hepatocellular carcinoma. Cancer Cell 2014; 25 (5): 666-681. PMID: 24768205.

10. Cao WJ, Wu HL, He BS, Zhang YS, Zhang ZY. Analysis of long noncoding RNA expression profiles in gastric cancer. World J Gastroenterol 2013; 19 (23): 3658-3664. PMID: 23801869. Central PMCID: 3691033.

11. Pang Q, Ge J, Shao Y, Sun W, Song $H$ et al. Increased expression of long intergenic non-coding RNA LINC00152 in gastric cancer and its clinical significance. Tumour B 2014; 35 (6): 5441-5447. PMID: 24523021.

12. Sun W, Wu Y, Yu X, Liu Y, Song H et al. Decreased expression of long noncoding RNA AC096655.1-002 in gastric cancer and its clinical significance. Tumour Biol 2013; 34 (5): 2697-26701. PMID: 23645148.

13. Yang F, Xue X, Zheng L, Bi J, Zhou $Y$ et al. Long non-coding RNA GHET1 promotes gastric carcinoma cell proliferation by increasing cMyc mRNA stability. FEBS J 2014; 281 (3): 802-813. PMID: 24397586.

14. Sun M, Xia R, Jin F, Xu T, Liu Z et al. Downregulated long noncoding RNA MEG3 is associated with poor prognosis and promotes cell proliferation in gastric cancer. Tumour Biol 2014; 35 (2): 1065-1073. PMID: 24006224.

15. Crick FH. The origin of the genetic code. J Mol Biol 1968; 38 (3): 367-379. PMID: 4887876.

16. Poliseno L, Salmena L, Zhang J, Carver B, Haveman WJ et al. A coding-independent function of gene and pseudogene mRNAs regulates tumour biology. Nature 2010; 465 (7301): 1033-1038. PMID: 20577206. Central PMCID: 3206313.

17. Sigova AA, Mullen AC, Molinie B, Gupta S, Orlando DA et al. Divergent transcription of long noncoding RNA/mRNA gene pairs in embryonic stem cells. Proc Nat Acad Sci USA 2013; 110 (8): 2876-2881. PMID: 23382218. Central PMCID: 3581948.

18. Bartel DP. MicroRNAs: target recognition and regulatory functions. Cell 2009; 136 (2): 215-233. PMID: 19167326. Central PMCID: 3794896.
19. Ventura A, Jacks T. MicroRNAs and cancer: short RNAs go a long way. Cell 2009; 136 (4): 586-591. PMID: 19239879. Central PMCID: 3910108 .

20. Hansen TB, Wiklund ED, Bramsen JB, Villadsen SB, Statham AL, Clark SJ et al. miRNA-dependent gene silencing involving Ago2mediated cleavage of a circular antisense RNA. EMBO J 2011; 30 (21): 4414-4422. PMID: 21964070. Central PMCID: 3230379.

21. Salmena L, Poliseno L, Tay Y, Kats L, Pandolfi PP. A ceRNA hypothesis: the Rosetta Stone of a hidden RNA language? Cell 2011; 146 (3): 353-358. PMID: 21802130. Central PMCID: 3235919.

22. Wang J, Liu X, Wu H, Ni P, Gu Z et al. CREB up-regulates long noncoding RNA, HULC expression through interaction with microRNA-372 in liver cancer. Nucl Acid Res 2010; 38 (16): 5366-5383. PMID: 20423907. Central PMCID: 2938198.

23. Lee JW. TM4SF5-mediated protein-protein networks and tumorigenic roles. BMB Rep 2014. PMID: 25027595.

24. Kwon S, Choi KC, Kim YE, Ha YW, Kim D et al. Monoclonal antibody targeting of the cell surface molecule TM4SF5 inhibits the growth of hepatocellular carcinoma. Cancer Res 2014; 74 (14): 3844-3856. PMID: 24802189.

25. Kwon S, Kim YE, Park JA, Kim DS, Kwon HJ et al. Therapeutic effect of a TM4SF5-specific peptide vaccine against colon cancer in a mouse model. BMB Rep 2014; 47 (4): 215-220. PMID: 24286311. Central PMCID: 4163885.

26. Wu YB, Huang YS, Xu YP, Sun YF, Yu DL et al. A high level of TM4SF5 is associated with human esophageal cancer progression and poor patient survival. Digest Dis Sci 2013; 58 (9): 2623-2633. PMID: 23633159.

27. Guo X, Xia J, Deng K. Long non-coding RNAs: emerging players in gastric cancer. Tumour Biol 2014. PMID: 25173641.

28. Lin XC, Zhu Y, Chen WB, Lin LW, Chen DH et al. Integrated analysis of long non-coding RNAs and mRNA expression profiles reveals the potential role of IncRNAs in gastric cancer pathogenesis. Intern J Oncol 2014; 45 (2): 619-628. PMID: 24819045.

29. Lee JW. TM4SF5-mediated protein-protein networks and tumorigenic roles. BMB Rep 2014; 47 (9): 483-487. PMID: 25027595.

30. Ryu J, Kang M, Lee MS, Kim HJ, Nam SH et al. Cross talk between the TM4SF5/focal adhesion kinase and the interleukin-6/STAT3 pathways promotes immune escape of human liver cancer cells. Mol Cell Biol 2014; 34 (16): 2946-2960. PMID: 24912675. Central PMCID: 4135608.

Received December 16, 2014. Accepted March, 12, 2015. 